\title{
Características del seguimiento de pacientes asmáticos que consultan por exacerbación en el Departamento de Emergencias de un hospital pediátrico
}

\author{
Characteristics of the follow-up pattern of asthmatic patients \\ who present with exacerbations in the Emergency \\ Department of a pediatric hospital
}

\author{
Mariela Centurión Duarte', Laura Godoy Sánchez²
}

\section{RESUMEN}

Introducción: El Asma es una de las enfermedades crónicas más frecuentes en pediatría, caracterizada por episodios recurrentes de sibilancias, disnea, opresión torácica y tos. Tiene un protocolo de tratamiento intercrisis (preventivo) que permite disminuir y controlar síntomas, mantener actividad física y una función pulmonar normal, así como prevenir las exacerbaciones. Objetivo: Describir la frecuencia del seguimiento y de tratamiento profiláctico de pacientes asmáticos de 6 a 12 años de edad que consultan en el Departamento de Emergencias del Hospital General Pediátrico Niños de Acosta Nu. Materiales y Métodos: estudio, observacional, descriptivo transversal, prospectivo. Fueron incluidos niños de 6 a 12 años de edad que consultaron en el Departamento de Emergencias de un Hospital Pediátrico con Diagnóstico de Asma. Se excluyeron pacientes con enfermedad pulmonar de base (EPOC, Fibrosis quística). Muestra probabilística de casos consecutivos. Variables: edad, sexo, procedencia, seguimiento del paciente asmático, causa de falta de seguimiento, Asma en la familia, clasificación de la Crisis de Asma, número de internaciones por Asma, tratamiento profiláctico. Los datos se analizaron en el sistema SPSS v21 utilizando estadística descriptiva. Se consideró un error alfa menor a 5\%. Resultados: $51.9 \%$ eran de sexo femenino, la edad media fue de $8,3 \pm 2$ años, casi la totalidad provenían de Gran Asunción, más de la mitad de los pacientes tuvieron crisis de asma leve, $48.9 \%$ con antecedentes familiares de Asma, 28.6\% tenían seguimiento por especialistas y $25.6 \%$ usaban algún tipo de medicación profiláctica. No se

\section{ABSTRACT}

Introduction: Asthma is one of the most frequent chronic diseases in pediatrics, characterized by recurrent episodes of wheezing, dyspnea, chest tightness and cough. It has an inter-crisis (preventive) treatment protocol that allows symptom reduction and control, thus maintaining physical activity levels and normal lung function, as well as preventing exacerbations. Objective: To describe the frequency of follow-up and prophylactic treatment of asthmatic patients from 6 to 12 years of age who presented to the Emergency Department of the Acosta Nu General Children'sHospital. Materials and Methods: This was an observational, descriptive, cross-sectional and prospective study. Children from 6 to 12 years of age who presented to the Emergency Department of a Pediatric Hospital and were diagnosed with Asthma were included. Patients with underlying lung disease (COPD, Cystic Fibrosis) were excluded. We used probabilistic sampling of consecutive cases. Variables were age, sex, place of residence, follow-up of the asthmatic patient, reason for lack of follow-up, asthma in the family, classification of the asthmatic crisis, number of hospitalizations for asthma, and prophylactic treatment. The data was analyzed in the SPSS v21 system using descriptive statistics. An alpha error of less than $5 \%$ was considered. Results: $51.9 \%$ were female, the mean age was $8.3 \pm 2$ years, almost all of the subjects came from the Greater Asunción area, more than half of the patients had mild asthma attacks, $48.9 \%$ had a family history of asthma, $28.6 \%$ were followed by specialists and $25.6 \%$ used some type of prophylactic medication. No relationship was observed between

${ }^{1}$ Universidad Católica Nuestra Señora de la Asunción. Asunción, Paraguay.

${ }^{2}$ Hospital General Pediátrico Niños de Acosta Nu. San Lorenzo, Paraguay.

Correspondencia: Laura Godoy Correo: legodoys@gmail.com

Conflicto de interés: Los autores declaran no poseer conflicto de interés.

Recibido: 26/06/2020 Aceptado:13/07/2020

Doi: https://doi.org/10.31698/ped.47022020009

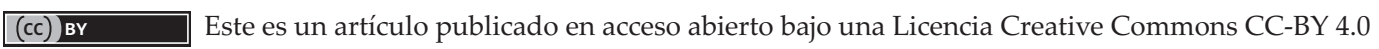


observó relación entre medicación profiláctica y número de internaciones. Conclusión: La cuarta parte de los pacientes tenía seguimiento y algún tipo de medicación profiláctica.

Palabras clave: Asma, niños, seguimiento, urgencias, profilaxis.

\section{INTRODUCCIÓN}

El Asma es una de las enfermedades crónicas, más frecuente en pediatría, caracterizada por episodios recurrentes de sibilancias, disnea, opresión torácica y tos. Adquiere una enorme repercusión no solo a nivel individual y familiar, sino también a nivel social y económico ${ }^{(1,2)}$.

El asma tiene un protocolo de tratamiento Inter crisis (preventivo) que permite disminuir las crisis, controlar los síntomas, mantener una actividad física y una función pulmonar normal, así como prevenir las exacerbaciones ${ }^{(2)}$.

El diagnóstico correcto del asma evita el exceso terapéutico ${ }^{(3)}$. Además, está claro que el costo que implica esta enfermedad, aumenta drásticamente a medida que disminuye el control de la misma, y, se podrían obtener ahorros sustanciales a través del manejo adecuado de los pacientes asmáticos ${ }^{(4)}$.

Si bien existen protocolos enfocados en el seguimiento de estos pacientes, la falta de cumplimiento influye en la evolución de la enfermedad. Las causas por las cuales los pacientes no realizan un tratamiento de sostén adecuada son multifactoriales. La insuficiente educación a los pacientes, la mala técnica de inhalación y la mala adherencia del tratamiento son algunas de ellas. Esta última es responsable del 38,8\% de la derivación de pacientes con Asma crónica. Además, juegan un papel importante la desinformación de los padres o encargados, negación del diagnóstico, y los factores socioeconómicos considerando el costo del tratamiento ${ }^{(5)}$.

En la población de niños con asma existen factores prophylactic medication and number of hospitalizations. Conclusion: A quarter of the patients had follow-up and some type of prophylactic medication.

Key words: Asthma, children, follow-up, emergencies, prophylaxis.

de riesgo de exacerbaciones de las crisis, entre los que se encuentran la atopia, la capacidad de incrementar la respuesta de la IgE a alergenos ambientales las infecciones respiratorias virales, el humo del cigarrillo, la polución del aire y las dietas ${ }^{(6)}$. El objetivo del presente trabajo fue describir las características del seguimiento y el uso de medicación profiláctica en pacientes asmáticos de 6 a 12 años de edad, de un hospital pediátrico de referencia.

\section{MATERIALES Y MÉTODOS}

Diseño y lugar del estudio: Estudio observacional descriptivo de asociación cruzada, prospectivo llevado a cabo en el departamento de Emergencias Pediátricas (DEP) del Hospital Pediátrico Niños de Acosta $\tilde{N u}$, en el periodo comprendido entre el 1 de mayo al 30 de setiembre del 2019.

Población y reclutamiento: por medio de un muestreo probabilístico de casos consecutivos, fueron incluidos en el estudio niños de 6 a 12 años de edad con diagnóstico de Asma que acudieron al DEP por exacerbaciones del cuadro (crisis asmática). Fueron excluidos los pacientes con otra enfermedad pulmonar de base, como EPOC o Fibrosis quística.

Se confeccionó un cuestionario con preguntas abiertas y cerradas, que los padres o encargados de los pacientes debían llenar previo consentimiento informado verbal.

Variables estudiadas: edad, sexo, procedencia, seguimiento del paciente asmático, causa de falta de seguimiento, antecedentes familiares de Asma, clasificación de la gravedad de la crisis asmática, 
internaciones por Asma, tratamiento preventivo, tipo de tratamiento preventivo.

\section{Definición de las variables}

Asma: enfermedad respiratoria crónica definida por una inflamación de las vías aéreas que se asocia con un aumento en la hiperreactividad de las vías aéreas que conduce a episodios recurrentes de sibilancias, disnea, opresión torácica y tos ${ }^{(1)}$.

Crisis asmática: empeoramiento agudo o subagudo de los síntomas y la función pulmonar del estado habitual del paciente ${ }^{(7)}$.

Clasificación de la severidad de la crisis: se utilizó el Pulmonary Index Score (PIS), que tiene en cuenta los siguientes parámetros: frecuencia respiratoria, sibilancias, relación Inspiración/Espiración, uso de músculos accesorios, saturación de oxígeno, considerando como crisis leve: PIS $<7$, moderada PIS: 7-11 y Grave: PIS >12.

Cálculo de tamaño de la muestra: para conseguir una precisión del $3 \%$ en la estimación de una proporción, mediante un intervalo de confianza del $95 \%$, con corrección para poblaciones finitas, asumiendo que, la proporción esperada es del $3 \%$ y que el tamaño de la población es de 110000, fue necesario incluir 133 pacientes.

\section{Procesamiento y análisis de los datos}

Los datos fueron analizados con SPSSv21 utilizando estadística descriptiva. Las variables cuantitativas se expresaron en medias con desvío estándar o en medianas según la distribución. Las cualitativas se expresaron en porcentajes. La asociación de variables cualitativas se realizó por la prueba de chi cuadrado. Se consideró un error alfa de $5 \%$.

El protocolo fue aprobado por el comité de ética de la institución con el consentimiento informado de los padres de los participantes.

\section{RESULTADOS}

Cumplieron los criterios de inclusión, 133 pacientes con diagnóstico de Asma, que consultaron en el DEP en el periodo de estudio. Las características demográficas, y la clasificación del Asma se observa en la Tabla 1.

Tabla 1. Datos demográficos y severidad de la crisis de pacientes asmáticos que consultaron en el Departamento de Emergencias del Hospital Pediátrico Niños de Acosta Nu. N=133

\begin{tabular}{lcc}
\hline Edad (años) & & \\
\hline Media (DE) & N & $8,3 \pm 2$ \\
Sexo & 64 & $\%$ \\
$\quad$ Masculino & 69 & 48.1 \\
$\quad$ Femenino & & 51.9 \\
Procedencia & 125 & \\
$\quad$ Asunción y Gran Asunción & 6 & 93.9 \\
$\quad$ Dpto. Central & 1 & 4.5 \\
$\quad$ Otros Dptos. & & 0.8 \\
Severidad de la crisis & 77 & 57.9 \\
$\quad$ Leve & 51 & 38.3 \\
$\quad$ Moderada & 5 & 3.8 \\
$\quad$ Grave & & \\
\hline
\end{tabular}

El 32,3\% (43/133) de los pacientes iniciaron seguimiento (controles periódicos), sin embargo el $6,9 \%(3 / 43)$ tenían un seguimiento irregular y el $4,6 \%(2 / 43)$ lo abandonaron después de un tiempo.

De los pacientes en seguimiento regular, $\mathrm{n}=38 \mathrm{el}$ $10,5 \%(4 / 38)$ no recibían la medicación en forma regular.

El 71,4\% (95/133) pacientes no tenían seguimiento. Respecto a los motivos que llevaron a la falta de seguimiento, se detallan en la Tabla 2.

Tabla 2. Causas que motivaron la falta de seguimiento de los pacientes según referencia de los padres o encargados, que consultaron en el Departamento de Emergencias del Hospital General Pediátrico Niños de Acosta Ñu. n=95

\begin{tabular}{lcc}
\hline Causas & $\mathbf{N}$ & $\mathbf{\%}$ \\
\hline Desconocimiento de la necesidad de seguimiento & $67(70.5)$ \\
No lo consideraban necesario & $16(17.8)$ \\
Las crisis eran poco frecuentes & $7(7.3)$ \\
Problemas con el turno & $3 \quad(3.1)$ \\
Falta de tiempo & $2 \quad(2.1)$ \\
\hline
\end{tabular}

Los antecedentes familiares de asma, el seguimiento con especialistas y los antecedentes de hospitalizaciones por exacerbaciones se observan en la Tabla3. 
Tabla 3. Antecedentes familiares de asma, seguimiento por especialistas y hospitalizaciones por exacerbación de los pacientes que consultaron en el Departamento de Emergencias del Hospital General Pediátrico Niños de Acosta Ñu. N=133

\begin{tabular}{lcc}
\hline Edad (años) & N & $\%$ \\
\hline Antecedentes familiares de Asma & & \\
SI & 65 & 48.9 \\
NO & & \\
Familiar con Asma & & \\
Materno & 30 & 22.6 \\
Paterno & 15 & 11.3 \\
Hermanos & 20 & 15 \\
Consulta con especialistas (Neumólogo, alergólogo) & & \\
SI & 38 & 28.5 \\
NO & 95 & 71.5 \\
Internaciones por Exacerbación & 77 & 57.9 \\
\hline
\end{tabular}

En cuanto al tratamiento profiláctico utilizado se describe en la Tabla 4. $(n=34)$

Tabla 4. Medicación Profiláctica utilizada por pacientes asmáticos que consultaron en el Departamento de Emergencias del Hospital General Pediátrico Niños de Acosta Nu. $(\mathrm{n}=34)$

\begin{tabular}{lcc}
\hline Medicación. & N & \% \\
\hline Corticoides Inhalado & 27 & 79.4 \\
Corticoides Inhalado más Montelukast & 3 & 8.8 \\
Montelukast & 1 & 2.9 \\
Otros & 3 & 8.8 \\
Total & $\mathbf{3 4}$ & \\
\hline
\end{tabular}

\section{DISCUSIÓN}

En el presente trabajo se observó que más de la mitad de los pacientes que consultaron en el Departamento de Emergencias del Hospital General Pediátrico Niño de Acosta Nu presentaron crisis asmática leve. Hubo predominio del sexo femenino. Estos datos coinciden en relación al sexo con lo reportado por Zapata Martínez y cols en el año 2008 en el Instituto Superior de Ciencias Médicas de La Habana en donde observaron que el $65.1 \%$ de los pacientes con asma eran mujeres ${ }^{(8)}$.

Referente al seguimiento se observó que 1 de cada 3 pacientes incluidos en el estudio, tenía seguimiento por especialistas (Neumólogo o Alergista), y que un tercio de los mismos recibían algún tipo de medicación profiláctica, esta cifra es inferior al 53,4
$\%$ sin seguimiento reportado por el estudio los de Zapata Martínez y cols ${ }^{(8)}$.

El asma, por su cronicidad y complejidad amerita un tratamiento integral, en el cual el conocimiento de médicos, pacientes y familiares sobre ella permitirá la concordancia, adherencia al tratamiento y seguimiento adecuado. En el presente estudio la falta de seguimiento de pacientes asmáticos, desde la perspectiva de sus padres o encargados, se debió a la falta de información acerca de la enfermedad. Coincidiendo con lo reportado por Kassise y cols quienes encontraron que el fracaso en el seguimiento de los pacientes asmáticos se relaciona a una educación sanitaria deficiente ${ }^{(9)}$.

Para el seguimiento de estos pacientes es necesario tener bien claras las características de esta enfermedad y los signos alarma. La valoración de los pacientes desde el punto de vista psicológico es muy importante porque influye en el reconocimiento de los síntomas, el manejo diario, al seguimiento y los resultados de la enfermedad ${ }^{(10)}$.

Por otro lado desde hace más de una década se conoce que la predisposición genética principalmente materna, junto con la exposición a alergenos son factores implicados en el desarrollo del asma ${ }^{(11)}$. En el presente estudio se encontró que casi la mitad de los participantes tenían antecedentes familiares de Asma, la mayoría maternos. Cifras cercanas a lo reportado por Berbesi y cols en Colombia, quienes encontraron que el $60 \%$ de los pacientes asmáticos tenían antecedentes familiares de asma ${ }^{(12)}$.

Más de la mitad de los pacientes que ingresaron al estudio, tenían antecedentes de internaciones por Asma, lo que podría deberse a la poca adherencia al tratamiento o la falta de información sobre la misma, lo que coincide con las Guías GINA para el Asma actualizado en el 2019, que refiere que más del $50 \%$ de los niños que padecen Asma no toman los medicamentos según se los recetó o la administración es incorrecta y recalca que la mala adherencia, contribuye a un control deficiente de los síntomas, a la aparición de exacerbaciones y al mayor riesgo de internaciones ${ }^{(7)}$. En este trabajo no se encontró relación entre profilaxis e internaciones. En un debate 
multidisciplinario realizado en España en el año 2016 encontraron que los factores desencadenantes del mal control del asma más frecuentes son: la insuficiente educación a los pacientes, la mala técnica de inhalación y la mala adherencia del tratamiento ${ }^{(5)}$. Por otro lado se conoce que aunque el tratamiento sea adecuado, muchas veces la respuesta al mismo puede variar debido a las diferentes variables genéticas reconocidas en el asma ${ }^{(13)}$.

Las medicaciones profilácticas más utilizadas fueron los corticoides inhalados, seguido de una terapia combinada entre corticoides inhalado y modificadores de leucotrienos (Montelukast), que coincide en parte con las guías GINA para el Asma, actualizado en 2019, que describe que los corticoides inhalados son los antiinflamatorios más eficaces para el asma, en cambio recalca que cuando una dosis intermedia de corticoides inhalado como monoterapia o terapia combinada con modificadores de leucotrienos, no logra un buen control del Asma la adición de un broncodilatador agonista $\beta 2$ de acción prolongada (formoterol, propionato de

\section{REFERENCIAS}

1. Callén Blecua M, Mora Gandarillas I. Manejo integral del asma. En: AEPap (ed.). Curso de Actualización Pediatría [Internet]. Madrid: Lúa Ediciones 3.0; 2017[citado el https://bit.ly/2JWgit2

2. Leonardo Cabello MT, Oceja-Setien E, García Higuera L, Cabero MJ, Pérez Belmonte E, Gómez-Acebo I. Evaluación de los conocimientos paternos sobre asma con el Newcastle Asthma Knowledge Questionnaire. Rev Pediatr Aten Primaria. 2013; 15(58):117-126. doi: http://dx.doi.org/10.4321/S1139-76322013000200003

3. García Merino A, Mora Gandarillas I. Diagnóstico del asma. Rev Pediatr Aten Primaria. 2013;(22):89-95. doi: http://dx.doi.org/10.4321/S1139-76322013000300010

4. Nunes C, Pereira AM, Morais-Almeida M. Asthma costs and social impact. Asthma Res Pract. 2017;3:1. doi: http://dx.doi.org/10.1186/s40733-016-0029-3

5. Plaza V, Quirce S, Delgado J, Martínez Moragón E, Pérez de Llano L. Debate multidisciplinar de expertos. Asma no fluticasona/salmeterol) al corticoide inhalado mejora los síntomas y la función pulmonar reduciendo las exacerbaciones en un mayor número de pacientes $\mathrm{y}$ más deprisa que la duplicación de la dosis del corticoides inhalado ${ }^{(7)}$.

Debido al enfoque del presente estudio, se considera una limitación, el hecho de que se evaluó el seguimiento de los pacientes, no así, la calidad de los mismos, así como tampoco se ha profundizado en las causas de la falta de seguimiento. Todos los pacientes asmáticos deberían tener un seguimiento a largo plazo, para tener mejores resultados.

\section{CONCLUSIÓN}

La cuarta parte de los pacientes tenía seguimiento y algún tipo de medicación profiláctica.

La gran mayoría de los pacientes tuvieron crisis asmáticas leves al ingreso al DEP. No se encontró relación entre medicación profiláctica y número de internaciones.

controlada: causas, consecuencias y posibles soluciones. Anales Sis San Navarra [Internet]. 2016[citado 10 jun 2019]; 39(3):357-370. Disponible en: http://scielo.isciii.es/ scielo.php?script=sci_arttext\&pid=S1137-66272016000 300003\&lng=es

6. Sánchez Tarrago N. Aspectos epidemiológicos del Asma: reseña. Reporte Técnico de Vigilancia [Internet]. 1996 [citado 03 junio 2019]. Disponible en : https://bit.ly/2QQrgkN

7. Global Initiative for Asthma (GINA). Global Strategy for Asthma Management and Prevention [Internet].GINA; 2019 [citado 10 jun. 2019]. Disponible en: http://www.ginasthma.com/

8. Zapata Martínez A, Salazar Domínguez LE, Cabrera Martell S, Carmona Serrano Y. Tratamiento profilactico del asma bronquial en dos consultorios Medicos. Rev Haban Cienc Méd [Internet]. 2008 [citado 12 sep. 2019]; 7(9). Disponible en: http://scielo.sld.cu/scielo.php?script =sci_arttext\&pid=S1729-519X2008000300016\&lng=es 
9. Kassise E, Ordaz R, Márquez C, Camperos N, Figueroa I. Educación y asma en la escuela: La carga del asma. Ausentismo y rendimiento escolar. Arch Venez Puer Ped [Internet]. 2010 [citado 12 sep. 2019]; 73(2):55-58. Disponible en: http://ve.scielo.org/scielo.php?script= sci_arttext\&pid=S0004-06492010000200008

10. Baiardini I, Sicuro F, Balbi F, Canonica G, Braido F. Psychological aspects in asthma: do psychological factors affect asthma management?. Asthma Res and Pract. 2015; 1(7). doi: http://dx.doi.org/10.1186/s40733-015-0007-1

11. Barrett EG. Maternal influence in the transmission of asthma susceptibility. Pulm Pharmacol Ther.
2008;21(3):474-84. doi: http://dx.doi.org/10.1016/j.pu pt.2007.06.005

12. Berbesí-Fernández DY, Segura-Cardona AM, GarcíaJaramillo M, Posada-Saldarriaga R. Seguimiento a una cohorte de niños con asma luego de una intervención familiar. Rev CES Med. 2013;27(2):143-152.

13. Vijverberg SJH, Farzan N, Slob EMA, Neerincx AH, Maitland-van der Zee AH. Treatment response heterogeneity in asthma: the role of genetic variation. Expert Rev Respir Med. 2018;12(1):55-65. doi: http://dx.doi.org/10.1080/17476348.2018.1403318 